\title{
LA LUDIEXPRESIÓN COMO ESTRATEGIA METODOLÓGICA EN LOS PROCESOS DE ENSEÑANZA EN NIÑOS DE PRIMER AÑO DE BÁSICA
}

\author{
Casa Erika ${ }^{1}$, Pozo Andrea ${ }^{2}$, García Ruth ${ }^{3}$ y Cando Edison ${ }^{4}$ \\ ms.educacion81incial@gmail.com¹, elizandy-ep@hotmail.com², ruth0277@hotmail.com³ \\ edisoncando@yahoo.es ${ }^{4}$ \\ https://orcid.org/0000-0001-7562-16781, https://orcid.org/0000-0003-0037-69752, \\ https://orcid.org/0000-0002-0044-2488 ${ }^{3}$,https://orcid.org/0000-0003-4467-1116 ${ }^{4}$ \\ Universidad Nacional de Rosario ${ }^{1},{ }^{2},-$ Ministerio de Educación ${ }^{1,2}, 3$, Universidad Central del Ecuador ${ }^{4}$ \\ Quito-Ecuador
}

Recibido (01/07/20), Aceptado (17/07/20)

\begin{abstract}
Resumen: Esta investigación tiene la finalidad de validar la aplicación de la ludiexpresión en los procesos de enseñanza-aprendizaje en el primer año de básica, en la educación particular de la ciudad de Quito, su análisis radica en la contribución que hará la lúdica en el desarrollo cognitivo e integral del niño. Mediante la investigación acción y el estudio de campo, procurando conocer como las maestras trasmiten los conocimientos y generan el progreso de las destrezas en los niños de este nivel. La recopilación de los datos se lo hizo mediante una metodología mixta, los colaboradores fueron 12 maestras de diversos niveles a las cuales se aplicó una encuesta en la que se pudo evidenciar la importancia del juego como una metodología para generar conocimientos en los niños de estas edades.
\end{abstract}

Palabras Clave: Lúdica, psicomotricidad, metodología, enseñanza-aprendizaje, desarrollo integral.

\section{LUDIEXPRESSION AS A METHODOLOGICAL STRATEGY IN THE TEACHING PROCESS IN CHILDREN OF FIRST YEAR OF ELEMENTARY SCHOOL}

Abstract: The purpose of this research is to validate the application of the Ludiexpression in the teaching learning process for the first year of basic in the private education of the Quito city; this analysis lies in the contribution that recreational activities will make in cognitive and integral development of the child, through action research and field study, trying to know how teachers transmit knowledge and generate the progress of skills in children of this level. Data was collected using mixed methodology, the collaborators were 12 teachers of different levels to whom a survey was applied, in which was evidenced the importance of the game as a methodology in order to bring about knowledge in children of those ages.

Keywords: Playful, psychomotricity, methodology, teaching-learning, integral development. 


\section{I.INTRODUCCIÓN}

La actividad lúdica es una experiencia ligada al descubrimiento mediante la cual los niños vivencian, conocen, inventan y comparten, logrando así comunicarse de manera múltiple [1]. Siendo esta una actividad innata del ser humano, se debe beneficiar a la educación mediante esta estrategia, favoreciendo de tal manera el conocimiento y aprendizaje. Al hablar de aprendizaje se considera como un proceso de creación de significados a partir de los conocimientos previos que trae cada niño, transformando así sus estructuras cognitivas [2].

El niño por su propia característica tiene al juego como parte de su desarrollo y su vivencia, esto hará que construya el conocimiento, por tal razón el juego y el aprendizaje debe apoyarse el uno en el otro.

Se evidencia en la actualidad la necesidad de recuperar la expresión lúdica en los procesos de enseñanza -aprendizaje en el primer año de educación básica, puesto que este acontecimiento no ha trascendido a pesar de que existe su vinculación a través del currículo del 2016 como macro referente [3]. La preocupación y priorización de las docentes radica en los aprendizajes cognitivos, escolarizando de manera radical a este nivel educativo apartando de esta manera al juego de sus actividades comunes.

La investigación recopilada pretende definir la importancia de la ludiexpresión como una estrategia metodológica y su influencia en la enseñanza - aprendizaje, explorando a profundidad sus características y la trascendencia en los niños de estas edades.

Este trabajo fundamenta sus resultados en un estudio transaccional o trasversal recolectando datos mediante la observación del fenómeno en su contexto natural "inédito" [4], señalando descriptivamente los hechos y eventos de la actualidad en forma sistemática y secuencial tomando en cuenta bibliografías sustentando así de manera teórica.

La recolección de la información se hizo mediante una encuesta aplicada a 12 docentes que integraban la institución particular Pensionado Rumiñahui, donde se elaboró esta investigación. Además, se realizaron actividades con los niños para valorar el juego y resaltar su relevancia.

Este trabajo está comprendido por cuatro secciones detalladas de esta manera; en la primera sección la importancia de la ludiexpresión y su relación con el proceso de enseñanza- aprendizaje, así como los beneficios en educación, la segunda expone la problemática del estudio en el área escolar del primer año de educación básica, en un tercer momento se evidencia la metodología utilizada y la última sección expone los resultados de este trabajo.

\section{II.DESARROLLO}

\section{A.Importancia de la lúdica}

La actividad lúdica es una necesidad innata, está presente durante toda la vida del ser humano consolidándose de esta manera en la evolución de la especie (filogénesis) [5]. En algunas ocasiones se observa a esta actividad como opuesta al trabajo, enfocando solo su finalidad en la diversión y el descanso, sin tomar en cuenta la trascendencia que tiene a nivel cultural, puesto que transmite valores, normas de comportamiento, resolución de problemas y esencialmente interviene en el desarrollo de la personalidad [5]. Pues es indispensable entender que jugar es una experiencia vinculada al placer que se expresa mediante múltiples lenguajes [1].

\section{B.Reseña histórica sobre el juego}

A lo largo de la historia el juego ha desempeñado varias funciones en el desarrollo del niño, considerándolo así desde la época de Platón y Aristóteles como formador de mentes [5]. Con el paso del tiempo en la segunda mitad del siglo XIX aparecen las primeras teorías del juego en las cuales se lo considera como el resultado de un exceso de energía acumulada [5]. En 1898 se concibe al juego como una preparación para las funciones de la vida adulta [5].

A inicios del siglo XX la lúdica empieza a evolucionar de la misma manera que la cultura humana, favoreciendo así a la capacidad de resolver problemas y a la vez como ayuda en el área de psicología [5].

La relación del juego y el desarrollo de los estadios cognitivos aparece con la teoría de Piaget pues de esta manera la educación empieza a relacionarse y abrir espacios, en el cual el individuo desarrolla sus capacidades sensorio motrices, simbólicas o de razonamiento, respetando su etapa evolutiva [1]. Es considerable entonces enfocar al juego desde el aspecto psicomotriz ya que ayuda al desarrollo de los sentidos, control muscular equilibrio, percepción y la capacidad de interiorizar el esquema corporal contribuyendo así al movimiento y por ende al desarrollo psicomotor completando así la maduración nerviosa [6].

\section{C.Relación juego, enseñanza- aprendizaje}

El aprendizaje por su lado se promueve en este mismo ambiente creativo y psicomotor, el cual debe estar proporcionado de estímulos que permitan intercambios de experiencias mediante el lenguaje corporal. [1]. Despertando así el interés del niño, lo cual es clave para crear espacios favorables para el proceso de enseñanzaaprendizaje.

Enseñar es una tarea que demanda esfuerzo por par- 
te del docente, pues se pretende lograr ajustes entre los contenidos que se va enseñar, las estrategias que se desea ampliar, las propuestas de experiencias de aprendizaje y las potencialidades que estarán disponibles por parte del niño [7]. Es entonces considerable poner atención en las diferentes formas de aprender respetando así los ritmos, intereses necesidades de cada niño y a la vez considerando sus estilos de aprendizaje de tal manera que marquen una experiencia significativa [2].

Si se habla de la importancia de atender las necesidades del infante, es entonces ineludible hablar sobre los procesos de enseñanza en el primer año de educación básica ya que este nivel está siendo marcado por la escolarización acarreando como consecuencias deserciones y falta de adaptabilidad a ese nuevo entono denominándola así sin retorno debido a que el niño se incorpora al sistema educativo [8].

\section{D.Articulación entre niveles}

Es preciso reincidir sobre la importancia de la articulación con el nivel inicial pues esta unión entre las partes ayudara a favorecer el aprendizaje de manera exitosa [9]. Creando espacios en los cuales el niño sea capaz de desarrollar habilidades metacognitivas, creativas y comunicacionales siendo este un requerimiento actual, entendiendo así al aprendizaje como un proceso de apropiación y edificación del conocimiento considerando al educador como un mediador que permita crear espacios de libertad para poder transmitir la forma de pensar fuera de lo convencional, es entonces cuando se debería priorizar una metodología establecida en el aprendizaje colaborativo [10]. Esta investigación del fenómeno de transición educativa entre los niveles escolares en los últimos años se ha incrementado a nivel mundial, preocupando de esta manera no solo el estado del niño, pues involucra la familia y los docentes [8].

\section{E.Metodología del juego}

Es así como esta investigación pretende combatir mediante el análisis este paradigma, sobre la educación del primer año de básica, ofreciendo como alternativa a una posible solución enseñar mediante el juego vinculando así el placer con el aprendizaje [1]. Deliberando que el jugar es un derecho del niño aprobado por la Asamblea General de las Naciones Unidas [1]. Pues su papel se destaca en proporcionar oportunidades para la expresión de la creatividad, imaginación, confianza en sí mismo, la autonomía y a la vez para desarrollar capacidades y aptitudes físicas, sociales, cognitivas y emocionales, pues así el niño edifica posición social dentro del mundo en el que se desenvuelve [11] .

En la actualidad la escuela nueva debe centrar el pro- ceso de enseñanza-aprendizaje en el niño, el ambiente, los materiales, intereses serán los medios para diseñar las prácticas [7]. El juego debe vivirse en cuatro momentos planificación, desarrollo, orden y evaluación [7]. Es importante detallar que el juego debe ser como la punta del iceberg por la cual la teoría pedagógica queda oculta, el niño no debe darse cuenta que está siendo educado si no pues la única finalidad debe ser jugar [7].

\section{F.Papel del docente en el proceso de enseñan- za-aprendizaje}

La función de los docentes ante una nueva situación de enseñanza debe ser facilitar y crear las vías necesarias para provocar experiencias que generen interés para captar la atención del estudiante, solo así él docente ganara ese territorio de manera significativa [12].

Lo que para el adulto puede parecer insignificante para el niño puede ser apasionante, la propuesta de enseñanza -aprendizaje no debe imponerse para causar en él sometimiento, por lo contrario debe causar intencionalidad para que de esta manera pueda apropiarse del conocimiento [7]. Pues, para que el niño tenga la intención de participar es indispensable darle motivos y es ahí donde se crea un desafío como docente pues se debe ocupar de dos campos la potencial significatividad de la propuesta y los aspectos motivacionales, aquí radica pensar en lo que se va enseñar y como se va hacerlo [13]. Es trascendente no caer en el error pues la actividad lúdica debe ser tratada como tal, no llamar juego a lo que no es ,como decir vamos jugar con los números cuando la intención es identificarlos, en muchas ocasiones se determina tales juegos para trabajar contenidos, cuando el niño aprecia la intención pierde su interés, pues el juego debe ser enseñado con el propósito de disfrute así se lo debe involucrar en el proceso pedagógico, la repetición constante del juego hará que el niño luego se ocupe del conocimiento que le proporciona ese juego, es decir si se invita un niño a jugar rayuela la intención debe ser disfrutar de jugar, después de varias vivencias con el mismo juego el niño pondrá atención en el resto e ira descubriendo si existen o no números y como están secuenciados es decir el juego debe provocar el aprendizaje [7].

La intencionalidad de aplicar esta estrategia metodológica es mejorar el proceso pedagógico pero a la vez ocuparse del desarrollo integral del niño favoreciendo en todos los campos en especial en el social en el cual debe aprender a cooperar, compartir, ayudar, solucionar su propios problemas obligándolo de tal manera a pensar y a respetar los criterios de los demás creando así lazos de amistad los mismos que le permitirán desarrollarse a posterior en su vida adulta [5]. 


\section{III.METODOLOGÍA}

La metodología utilizada en este trabajo es de condición probabilístico intencional, está formada por 12 docentes del centro educativo particular Pensionado Rumiñahui, ubicada en el sector norte de la ciudad de Quito, de las cuales el 66.66\% son docentes de diferentes niveles y el 33,33\% de especialidad, todas de género femenino.

El instrumento utilizado fue una encuesta analítica basada en un conjunto de preguntas que estuvo conformados por 15 ítems, en una escala de tipo Likert de 4 alternativas de respuesta. La encuesta está conformada por 4 factores. El primer factor compuesto por aspectos relacionados con la lúdica y el proceso de enseñanzaaprendizaje, el segundo aspecto relacionado con el desarrollo psicológico e integral del niño de primer año de educación básica, el tercer factor la importancia de la psicomotricidad y su vinculación con la enseñanza y finalmente el aspecto favorable en la aplicación lúdica como estrategia metodológica.

La información recopilada se coordinó con la autoridad de la institución educativa, de la misma manera para la aplicación del instrumento medición documental contribuyeron las docentes de los diferentes niveles de pre -escolar a séptimo año de educación general básica y de especialidad, los mismos que fueron aplicados de forma individual y voluntaria. Igualmente se informó a los participantes sobre la finalidad de la investigación y de la prudencia en el manejo de los datos obtenidos.

\section{IV.RESULTADOS}

En general como se había expuesto en la primera sección sobre la importancia de la lúdica en la educación, los resultados de la encuesta, demostraron valores estadísticos positivos, pues la mayoría de docentes que integraban la institución, consideraron la importancia de aplicar la ludiexpresión como estrategia metodológica, para mejorar el proceso de enseñanza -aprendizaje, en el niño de primer año de educación general básica, considerando así la posibilidad de anular la escolarización en este nivel y favorecer de esta manera el desarrollo integral, esta propuesta permitió a las docentes concienciar sobre el interés del niño, la experiencia significativa, el nivel emocional y la capacidad de desarrollar el área cognitiva en el escolar. Sin embargo, hay un porcentaje mínimo que no considera muy relevante la aplicación de dicho método.

De la misma manera el efecto positivo puede dar paso a una elaboración de una guía didáctica basada en la metodología lúdica para docentes de este nivel.

Por otro lado, al beneficiar a los niños con esta estrategia, los padres de familia alejaran el grado de preocu- pación por la deserción escolar y el estrés de sus hijos en estas edades.

Al procesar e indagar la información se encauzo todo para llevarlo a figuras comparando así sus resultados. A continuación, se presentan algunas de estas preguntas desarrolladas en la encuesta que marcaron efectos representativos.

Se puede evidenciar en un alto porcentaje la relación que debe existir entre el juego y la psicomotricidad, aun sus objetivos sean diferentes, pues la lúdica busca la satisfacción corporal y mental mientras que la psicomotricidad se relaciona con su entorno, mediante el esquema corporal a través del movimiento. Pero sin duda la dos contribuirán al proceso de enseñanza- aprendizaje y de tal manera cooperarán al desarrollo integral en el niño.

Un importante porcentaje de docentes consideran que el niño a través de su entorno, tiene la capacidad de vivir experiencias significativas, las cuales le ayudaran a integrar los aprendizajes que perduraran a lo largo de su vida, puesto que parten de la práctica propia.

Se puede evidenciar que la gran mayoría de docentes consideran que la estimulación faculta las redes neuronales en los niños, provocando así la asimilación del nuevo conocimiento desarrollando la inteligencia y el lenguaje.

Según el criterio de la mayoría de docentes, consideran que la lúdica permite liberar tensiones y despiertan el interés por aprender, basado en este criterio se puede pensar que, al cubrir las necesidades del educando, desarrollará sus propias potencialidades.

La psicomotricidad por su parte aporta en el desarrollo de los aspectos cognitivos, socio afectivos y motrices ocupándose de manera íntegra en el niño. Un porcentaje alto maestras coinciden que, al ocuparse de todos los aspectos mencionados, se está cubriendo con las necesidades y por ende facilitara el proceso de enseñanza- aprendizaje.

En un porcentaje alto las maestras consideran que la experiencia significativa le permite al niño vivenciar, interiorizar, transformar y desarrollar su psicomotricidad.

Las preguntas enfocadas en relación a la lúdica, psicomotricidad y procesos de enseñanza - aprendizaje, logran dar una pauta hacia un cambio de visión en la formación pedagógica del primer año de básica, pues su aplicación contribuirá al mejoramiento en la educación de nuestro país, respetando así los derechos y la libertad del niño.

\section{V.CONCLUSIONES}

Los resultados obtenidos mediante la encuesta revelan que al utilizar la ludiexpresion como una estrategia 
metodológica los procesos de enseñanza aprendizaje mejoraran de manera primordial, pues el protagonista en la educación será él estudiante y por ende su interés se verá reflejado al momento de aprender. Comprendiendo así que jugar es una actividad multidimensional de cada ser humano pues integra afectividad, corporalidad, pensamiento y movimiento nivelando así los nuevos conocimientos de manera creativa y lúdica.

$\mathrm{Al}$ acompañar a las docentes en este proceso, permitió que reflexionen sobre su metodología de trabajo escolarizado y los efectos que están provocando en los niños de primer año, entonces se interesan por aplicar una metodología que facilite los procesos cognitivos y a la vez genere placer en el momento de educar creando así espacios comunicativos idóneos entre el niño y la docente.

La posibilidad de utilizar la metodología lúdica como una propuesta pedagógica permitirá reconocer a las docentes que su aplicación ayudará a su función posibilitando los espacios educativos más libres, placenteros y significativos. De esta manera la educación escolarizada pasara a la historia convirtiéndose en activa y recreativa. La indagación sobre esta temática da paso a otro espacio de estudio en el desarrollo del niño, pues el juego cubre distintas áreas de interés, comprendiendo así el desarrollo socioemocional importante también en el proceso de aprendizaje y su relación con las neurofunciones.

\section{REFERENCIAS}

[1]L. Pitluk, La centralidad del juego en la educación inicial, Santa Fe - Argentina: Homosapiens, 2019, pp. 18,19 .

[2]F. A, M. I. Cabrera, Rodriguez y A. Bordas , «Estrategias de evaluacion de los aprendizajes centradas en el proceso,» Revista Española de Pedagogía, vol. 59, $\mathrm{n}^{\circ}$ 218, pp. 25-48, enero-abril 2001.

[3]M. d. Educación, Currículos de los niveles de educación obligatoria, Subnivel preparatoria, Quito, Pichincha: MInisterio de Educacion, 2019.

[4]E. S. Casa, Llano, "La luediexpresión como estrategia metodológica en los procesos de enseñanza-aprendizaje",tesis previo a optar el título de maestria, Quito: Universidad Central del Ecuador, 2010.

[5]J. Montañez, M. Parra, T. Sánchez, R. López, J. M. Latorre, P. Blane, M. J. Sánchez, J. P. Serrano y P. Túregano, «El juego en el medio escolar,» Facultad de Albacete, $\mathrm{n}^{\circ} 15$, pp. 235-260, 2000.

[6]J. G. Lopez, Araujo , A. E. Pozo, Potosi, Y. C. Bodero, Aguayo y N. J. Loor , Aguayo, «El juego en el desarrollo intelectual del niño,» Universidad Ciencia y Tecnología, vol. 1, nº 1, pp. 97-106, 2020.
[7]Sarle y P. M. Sarlé, Lo importante es jugar, Santa feArgentina: Homosapiens, 2016.

[8] A. Castro, P. Ezquerra y J. Argos , «La transición entre la escuela de educación infantil y la educación primaria: prespectivas de niños, familia y profesorado,» Revista española de pedagogía, vol. 70, n 253 , pp. 537 552, 2012.

[9]E. Luchetti, Articulación, Buenos aires- Argentina: Bonum, 2012, pp. 11-12.

[10]A. García, Valcarél, V. Basilotta y C. López, , «Las tics en el prendizaje colaborativo en el aula de primaria y secundaria,» Revista cientifica de comunicación y educación, vol. XXI, n ${ }^{\circ}$ 42, pp. 65-74, enero 2014.

[11]J. A. Gallardo, López y P. Gallardo, Vázquez , «Teorías sobre el juego y su importacia como recurso educativo para el desarrollo integral infantil,» Revista educactiva digital hekademos, $\mathrm{n}^{\circ}$ 24, pp. 41-51, 2018.

[12]J. E. Mendoza, Carrera , E. G. Montero, Zamora , G. J. Castro, Castillo y D. J. Burgos Medieta, «Las artes y su incidencia en todos los niveles educativos de la univesridad estatal de milagro,» Revista, Universidad, ciencia y tecnología, vol. $2, \mathrm{n}^{\mathrm{o}} 2$, pp. 63-67, noviembre 2019.

[13]R. García Molina , «Ciencia recreativa: un recurso didáctico para enseñar deleitando,» Revista eureka sobre enseñanza y divulgación de las ciencias, vol. $8, \mathrm{n}^{\circ}$ extraordinario, pp. 370-392, noviembre 2011.

\section{RESUMEN CURRICULAR}

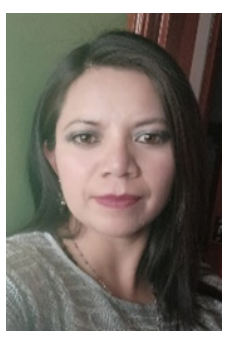

Pozo Potosi, Andrea Elizabeth; Magister en Educación y Proyectos de Desarrollo con Enfoque de Género, Licenciada en Ciencias de la Educación mención Profesora Parvularia, de nacionalidad ecuatoriana, docente del nivel Inicial en el Ministerio de Educación, apasionada de la lectura y la investigación. Cursando el Doctorado en Ciencias de la Educación en la Universidad Nacional de Rosario - Argentina.

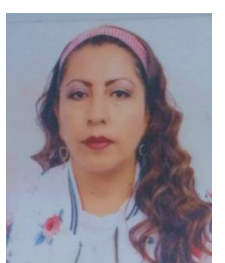

García, Ruth; Magister en Pedagogía de nacionalidad ecuatoriana, en la actualidad directivo de una institución educativa emblemática del sistema educativo fiscal gracias a su conocimiento y título en licenciatura con mención en gerencia y liderazgo educativo, amante de la lectura e investigación de las neurociencias y procesos educativos, razón por la cual su título inaugural lo obtuvo en el instituto superior Normal Manuela Cañizares con alusión en docencia de educación básica. En este momento se encuentra cursando el Doctorado en Ciencias de la Educación en la Universidad Nacional de Rosario Argentina. 


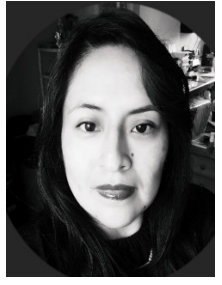

Casa Llano Erika Silvia; Magister en Educación Inicial, Licenciada en Ciencias de la Educación mención Profesora Parvularia, nacionalidad ecuatoriana, en la actualidad directivo de un Centro de Desarrollo Para la Primera Infancia Ministerio de Educación, su amor por la enseñanza en la educación Inicial, le ha motivado día a día para buscar siempre cambios e innovación en su área. Actualmente se encuentra cursando un Doctorado en Ciencias de la Educación en la Universidad Nacional de Rosario Argentina. https:/casa-erika.com/

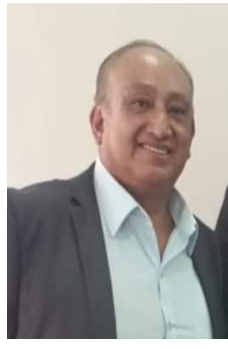

Cando Vaca, Edison Franklin; Licenciado en Artes en la Universidad Central del Ecuador. Maestría en Gerencia de Proyectos Educativos y Sociales en la Universidad Central del Ecuador. Docente investigador de la Facultad de Filosofia de la Universidad Central del Ecuador. edisoncando@yahoo.es 